Gesnerus 60 (2003) 62-82

\title{
Un règlement pour lutter contre la peste
}

Genève face à la grande peste de Marseille (1720-1723)

Alexandre Wenger

\section{Summary}

In 1721, when Geneva is threatened by a terrible epidemic devastating the south of France, the physician Jean-Jaques Manget states that "The Magistrates must establish a good Policy in time of plague"; and asks himself how it is possible to carry on "without good Regulations?". The State Archives of Geneva hold a draft consisting of 72 articles concerning health policy meant to be applied in case of plague. This is a remarkable document that enables an overview of the city's organisation in times of plague; it also allows to analyse some of the medical and sanitary theories this organisation is based on.

\section{Résumé}

«[L]es Magistrats sont obligez d'établir une bonne Police dans les Villes en temps de Peste»; et comment y parvenir «sans de bons Réglemens?», s'inquiète le médecin genevois Jean-Jaques Manget en $1721^{1}$, alors que sa ville est en crise, menacée par une terrible épidémie qui ravage le sud de la France. Les Archives d'Etat de Genève ont conservé un projet de règlement, composé de 72 articles de police sanitaire destinés à être appliqués dans le cas où la cité serait frappée par le fléau. Ce document constitue un remarquable outil pour appréhender tant l'organisation de la cité face à la menace de peste que, le cas échéant, l'influence de certaines théories médicales et sanitaires sur cette organisation.

1 Manget 1721, t. 1, 57.

Alexandre Wenger, Institut universitaire romand d'histoire de la médecine et de la santé, CMU - Case postale, CH-1211 Genève 4 (wenger3@etu.unige.ch). 
Depuis sa réapparition en Europe, au milieu du $\mathrm{XIV}^{\mathrm{e}}$ siècle, la peste a sévi de nombreuses fois à Genève. Entre 1450 et 1550, époque à partir de laquelle les archives permettent une appréciation détaillée de la situation, on dénombre quatorze résurgences du fléau s'étendant sur un total de vingt et un ans. Dès la seconde moitié du XVI ${ }^{\mathrm{e}}$ siècle, avec l'instauration d'un registre des morts qui constate officiellement les décès et mentionne leurs causes, une quantification des pertes humaines dues à la peste devient possible. Ainsi, les six retours de la maladie, qui s'étendent entre la grave épidémie de 1568-1572 et celle de 1636-1640, la dernière à frapper Genève, causeront la mort d'environ 6500 personnes, soit $14,5 \%$ du total des décès ${ }^{2}$.

Mais l'histoire de la peste à Genève ne s'arrête pas en 1640; les rumeurs de contagion en provenance de contrées plus ou moins lointaines sont fréquentes et les registres des Conseils genevois se font l'écho d'alarmes récurrentes jusqu'à la fin du XVIII ${ }^{\mathrm{e}}$ siècle. Entre 1720 et 1723, la maladie se manifeste une dernière fois en Europe occidentale sous une forme extrêmement virulente. Malgré une police maritime stricte, la peste est introduite à Marseille en mai 1720 par le Grand-Saint-Antoine, navire marchand français en provenance de Syrie. Les autorités municipales, soucieuses de leur commerce, ne réagiront officiellement que le 23 juillet 1720 , après plusieurs morts indubitablement dues à la peste 3 . C'est grâce à l'effort unificateur de l'Etat français que l'épidémie restera cantonnée dans le sud du pays: confirmant les velléités absolutistes et centralisatrices déclarées au siècle précédent, c'est en effet la Régence, et non plus les parlements régionaux, qui pour la première fois supervisera le gigantesque cordon sanitaire isolant les contrées infectées. L'épidémie fera tout de même près de 120000 morts, soit près d'un tiers de la population provençale de l'époque.

Genève est avisée d'une «possible contagion à Marseille» le 31 juillet 1720, par un courrier de l'intendance savoyarde à Chambéry ${ }^{4}$ Le Petit Conseil et la Chambre de la Santé décident immédiatement la mise en place d'un panel de mesures concrètes déjà déployées lors des épidémies précédentes: la ville est «purifiée» de ses mendiants, la surveillance aux portes est renforcée et les personnes et marchandises en provenance de contrées suspectes doivent dorénavant être munies de bullettes de santé6. Dans le courant du mois d'août 1720, la péjoration de la situation dans le sud de la France impose l'idée d'une crise appelée à durer, en conséquence de quoi la déci-

\footnotetext{
2 Perrenoud 1979, 446-448.

3 Cf. Gaffarel 1911.

4 Aeg-RC n ${ }^{\circ} 219,295$.

5 Wenger 2001.

6 Aeg-RC n 219, 306-309.
} 
sion est arrêtée de construire deux lazarets de quarantaine, l'un à Châtelaine pour les marchandises ${ }^{7}$, l'autre à Sécheron pour les personnes ${ }^{8}$. Ces établissements ont une fonction tant sanitaire que politique et publicitaire: il s'agit en effet de fournir de solides garanties prophylactiques pour ne pas s'exposer à une interdiction de commerce de la part des Suisses, eux-mêmes en butte aux pressions de leurs partenaires commerciaux situés plus au nord, et par conséquent plus loin du foyer épidémique. La situation de Genève, point nodal entre les deux marchés que sont, d'une part, les cantons helvétiques et, d'autre part, la vallée du Rhône, tient d'un équilibre économique, politique et sanitaire ténu en tant de peste.

Fin 1721, les Commis sur la Santé planchent sur un règlement destiné à régir la ville et la campagne genevoises au cas où la peste la toucherait effectivement. Ce Projet d'un Règlement général pour l'ordre qui devra être observé en cette Ville, au cas qu'elle vint à être affligée de la Peste ${ }^{9}$, dont la présentation fait l'objet de cet article, unique exemplaire conservé par les Archives d'Etat de Genève, reste, bien qu'elle soit très complète, une mouture provisoire. Imprimé en décembre 1721, ce document devait être envoyé aux alliés bernois à titre consultatif. Mais son intérêt s'estompera lorsque, dans les premiers mois de 1722, la menace provençale reculera. Le 2 octobre 1722, une lettre circulaire, avec Berne et Fribourg, proclamera la désinfection de Marseille et la levée progressive des barrières sanitaires ${ }^{10}$. Ce ne sera toutefois qu'en été 1723 que le rétablissement intégral de la libre circulation des personnes et des marchandises permettra de définitivement fermer les lazarets ${ }^{11}$.

L'établissement de textes qui codifient les comportements en temps de peste est une constante depuis le retour de l'épidémie en Europe au XIV siècle ${ }^{12}$. L'histoire de tels documents s'inscrit dans la longue durée; proposant d'abord des mesures locales plus ou moins disparates (encore que toutes de nature spirituelle, morale ou policière), leur contenu aura tendance à s'homogénéiser au fil des siècles. D'une municipalité à l'autre, d'une région à l'autre ou d'une nation à l'autre ${ }^{13}$, les règlements sont copiés, développés

\footnotetext{
7 Aeg $-\mathrm{RC} \mathrm{n}^{\circ} 219,353 \& 362$.

8 Aeg - Santé A5, 44.

$9 \mathrm{Aeg}-\mathrm{PH} 4455$, pièce $\mathrm{n}^{\circ} 9$ - cf. fig. 1 et la transcription intégrale de ce document en annexe.

10 Aeg $-\mathrm{RC} \mathrm{n}^{\circ} 221,467,493 \& 507$.

11 Aeg - Santé A7, 87 \& 92.

12 C'est le cas par exemple de l'Ordinamenta sanitatis tempore mortatis de la ville de Pistoie (Carpentier 1962,131-133).

13 Par exemple, en 1577, le Collège Royal de Londres dresse une liste des précautions à observer en cas de peste: ce texte, établi sur la base de documents italiens et français antérieurs est inclus en 1578 dans un recueil de décrets puis réimprimé sans modifications en 1592,1593, 1603,1625 (Cipolla 1992, 17; Slack 1985).
} 


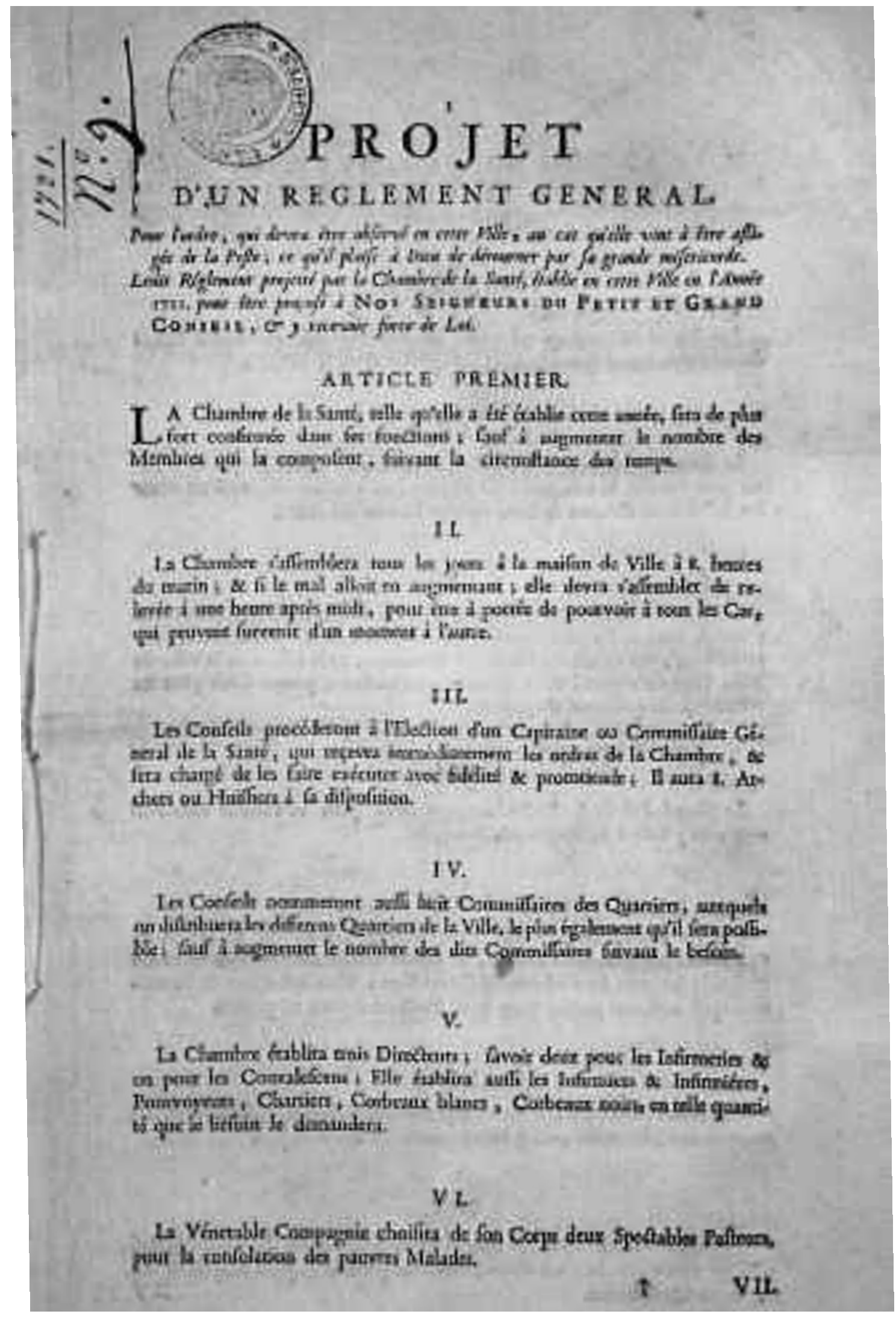

Fig. 1. Archives d'Etat de Genève, PH 4455 pièce $n^{\circ} 9$. 
à chaque retour d'épidémie et progressivement coordonnés quant à leur action; la réglementation en temps de peste est donc liée à la création d'organismes municipaux spécifiquement destinés à la préservation de l'hygiène publique. Les principes fondamentaux qui régiront les grandes mesures collectives engagées contre la peste au XVIII ${ }^{e}$ et $\mathrm{XIX}^{\mathrm{e}}$ siècle sont en place dès la première moitié du XVII ${ }^{\mathrm{e}}$ siècle en $\mathrm{Italie}^{14}$, et dès la seconde moitié du XVII ${ }^{\mathrm{e}}$ siècle plus au Nord; pour Berne et le Pays de Vaud, devant les menaces réitérées de l'épidémie - en particulier celle qui décime l'Oberland bernois en 1668-1669 - les règlements, qui jusqu'alors présentaient une forme analogue aux «mandats sur les mœurs $»^{15}$, intègrent progressivement des mesures pratiques de restriction des déplacements des personnes, de limitation du trafic commercial et d'instauration de bullettes de santé, ainsi que les sanctions en cas de non-respect de ces mesures ${ }^{16}$. Pour l'Italie, et particulièrement le quadrilatère Venise, Milan, Gênes, Florence, que Carlo Maria Cipolla estime être «en avance ${ }^{17}$ en matière d'organisation contre la peste, l'évolution générale est la suivante: fin $\mathrm{XV}^{\mathrm{e}}$ siècle, les magistratures de santé, quelle qu'en soit la forme administrative locale, sont des institutions d'urgence. Fin $\mathrm{XVI}^{\mathrm{e}}$ siècle, elles deviennent progressivement permanentes, avec une organisation hiérarchisée: des magistratures de santé centralisées établies dans les capitales des différents Etats président à l'établissement de magistratures d'urgence en cas de peste dans les villes de moindre importance. Leur rôle est par ailleurs non seulement de lutter contre la peste en temps de peste, mais de garantir l'hygiène urbaine en temps d'accalmie ${ }^{18}$. Au niveau européen, l'acceptation des règlements au sujet de la peste sous forme de règles sanitaires par les gouvernements se fait essentiellement au $\mathrm{XVIII}^{\mathrm{e}}$ siècle et dans la première moitié du $\mathrm{XIX}^{\mathrm{e}}$ siècle $^{19}$.

14 Cipolla 1992, 113.

15 Olivier 1939a, 607

16 Olivier 1939a, 608.

17 Cipolla 1992, 16.

18 Cipolla 1992,15-16.

19 Biraben 1975, t. 2, 103-104. En ce qui concerne les règlements maritimes, on peut consulter la reproduction dans l'ouvrage de Daniel Panzac d'une «Instruction pour les intendants de la Santé sur les usages et coutumes du Bureau de Marseille (1716)» (Panzac 1986, 127-129), document riche qui offre une base de comparaison avec le règlement de Genève. Bien qu'elle porte sur les problèmes liés au commerce maritime, et qu'elle vise en particulier à contrôler celui en provenance du Levant, cette réglementation se fonde sur les mêmes principes «hygiéniques» que celle de Genève. Pour constituer ce document, les Intendants ont recueilli «dans les anciennes Délibérations les Usages qu'on a observé, où chacun trouvera dequoy s'instruire par soi-même des différentes fonctions qu'il est obligé de remplir» (Panzac 1986, 139), comme quoi les anciens mesures restent toujours la principale source d'inspiration des règlements. 
Les documents administratifs genevois les plus anciens concernant la peste sont deux règlements pour l'hôpital des pestiférés de Plainpalais remontant respectivement à la fin du $\mathrm{XV}^{\mathrm{e}}$ siècle et à $1503^{20}$. On y trouve sous forme embryonnaire des dispositions financières, administratives, sanitaires et religieuses appelées à être développées au cours des épidémies à venir. Léon Gautier a publié les règlements relatifs à la peste de 1564,1568 et 1571, ainsi que celui de 1629 qui n'est qu'une reprise, avec de légères modifications, de celui de $1615^{21}$. Consulter les registres tenus au cours des épidémies précédentes pour se remémorer les mesures prises est une pratique constante, presque un réflexe, de la part des autorités. Le $1^{\text {er }}$ mars 1721 , en tête du registre de la Chambre de la Santé, le «Reiglement au fait de la Contagion observée à Genève en l'an 1636 sous l'aveu de nos très honorés Seigneurs», en 53 articles, est méticuleusement recopié22. Le passé devient une ressource lorsque gronde la peste ${ }^{23}$.

Le Projet d'un Règlement de 1721 est intéressant - et, dans un sens, très novateur - à plus d'un titre. Ne comptant pas moins de 72 articles, il est d'abord le document de son espèce le plus complet jamais imprimé à Genève. Par ailleurs, anticipant les conséquences potentielles de l'arrivée du fléau, il est le seul à avoir été élaboré dans une perspective entièrement prévisionnelle ${ }^{24}$; à ce titre, il est un témoin privilégié de l'empirie et de la théorie fondant l'action sanitaire de son époque. Enfin, son élaboration a fait l'objet d'une concertation - qui relève tant de la collaboration que de la surveillance mutuelle - avec Berne qui s'attelait alors à l'élaboration d'un corps de lois analogue 25 .

Le contrôle est le maître-objectif d'un tel règlement (ce que confirme son intitulé): la peste constitue un état d'exception, une situation de crise, qu'en l'absence d'une thérapie miracle on ne peut endiguer que par une

20 Chaponnière/Sordet 1844, 451-455.

21 Gautier 1905, 591 ss.

22 Aeg-Santé A6,1-10.

23 La convocation de mesures passées est une constante (cf. note 19): d'anciens règlements sont repris, mais également d'autres types de documents. C'est le cas du gouvernement bernois pendant la peste de Marseille; il renseigne «le public par deux brochures, simples reproductions d'écrits déjà vieux, le Consilium medicum über die Leydige Pest, de 1629, 28 p. du médecin de la ville David König, et le Summarischer Bericht und einfaltiger Denck-Zettul, de 1667, 10 p.» (Olivier 1939b, 620).

24 A Lausanne, le principal document de cette période est un règlement de septembre 1720, modifié et précisé par la suite à plusieurs reprises en fonction des rumeurs en provenance de la Provence (Olivier 1939b, 621-623). Ce règlement comporte les mesures habituelles de police, d'ostracisme, de désinfection, etc. - l'orientation du Règlement genevois est différente, clairement d'anticipation.

25 Le 17 janvier 1722, Genève reçoit le Projet de Reglement du Conseil de Santé de la ville de Berne, en Cas de Contagion (Aeg - PH 4455, pièce $\mathrm{n}^{\circ} 10$ ). 
police et une discipline sociale fortes. Les articles le composant peuvent être regroupés en cinq ensembles: ceux qui ont trait aux fonctions et pouvoirs de la Chambre de la Santé. Ceux qui concernent les bâtiments d'isolement. Ceux qui donnent les prescriptions relatives au personnel soignant, y compris les corbeaux et les enterreurs. Ceux qui touchent à la distribution des moyens de subsistance et, finalement, ceux qui regardent le contrôle et le dénombrement des malades, des morts et des convalescents. A cela s'ajoute encore l'article LXXIII, sorte d'appendice final qui fait le point sur les provisions dont la ville doit se pourvoir. Ce dernier article est le seul à ne pas anticiper l'arrivée du fléau: il est le reflet de l'achalandage de Genève en décembre 1721. On y trouve d'ailleurs le seul renvoi à l'inquiétude qui devait être celle de la population de l'époque: les particuliers se sont fendus de «provisions extraordinaires» $\left(\right.$ point $2^{\circ}$ ).

Le Projet de règlement permet de se faire une image précise du système de cabanons destinés, en cas de peste, à isoler les infects, les suspects et les convalescents. Essentiellement édifiés sur la jonction entre l'Arve et le Rhône et sur Plainpalais, c'est toute une topographie de ces lieux d'isolement qui se dessine autour des murs de la ville. Les cabanes seraient numérotées (LIV) et incluses, en fonction des personnes qu'elles seraient appelées à recevoir, dans un espace ceint de barrières, avec toujours une sortie unique et gardée (XLV). Ainsi, les infirmeries pour les pauvres occuperaient un de ces espaces (IX), un autre étant dévolu à celles des «gens moyennez» (X). Deux autres parcelles reviendraient respectivement aux convalescents pauvres (XI) et aux convalescents riches (XII). Les cabanes de quarantaines auraient leurs emplacements, pour les démunis (XIII) comme pour les nantis (XIV). Il faut ajouter à ce tableau une maison destinée à loger les infirmiers des malades (XVII) et une autre pour l'hébergement des infirmiers des convalescents (XVII). Les corbeaux résideraient dans des capites jouxtant le cimetière autour de l'ancien hôpital des pestiférés (XVIII) tandis que la soldatesque pourrait camper dans les prés et les fossés comblés alentour (XIX).

La Chambre de la Santé, dont les pouvoirs seraient étendus (XXIX), veillerait à la gestion de l'ensemble; son outil principal serait l'hôpital général du Bourg-de-Four, réelle centrale de distribution et de contrôle des vivres et des médicaments (XLVIII). Sur le rapport fait par les médecins, cet établissement enverrait chaque jour aux barrières des divers «enclos sanitaires» de quoi en nourrir les occupants (XLIX).

Le traitement des gens «moyennez» et des pauvres représente la principale disparité entre le règlement de Genève et celui de Berne. Comme le reconnaît M. Fischer de Reichenbach, président du conseil de santé de la ville de Berne dans une lettre de décembre 1721, les infirmeries bernoises ne 
concernent que «ceux qui ne seront pas en Etât de rester chès eux, en un mot, $[\ldots]$ les pauvres ${ }^{26}$; les gens fortunés ont l'autorisation, sous certaines conditions, de faire la quarantaine calfeutrés à leur domicile, et cela même s'ils sont suspects. Au demeurant, les vues des deux cités sont majoritairement partagées, depuis les durées de séquestre, qui sont identiques, jusqu'aux modalités de désinfection et aux mesures d'ostracisme.

Trois principes fondamentaux régissent le dispositif genevois. Il y a d'abord la séparation. Elle constitue le plus petit commun dénominateur de tout acte de lutte contre la peste: séparer les habitants, cloisonner le risque, c'est se donner la possibilité de le contrôler. Ce principe préside à l'identification des maisons infectes (XXIII) et à l'attribution de signes distinctifs au personnel soignant - marques de couleurs (XXXIII), clochettes (XXXIV) ou baguettes blanches (LVII) selon les cas. C'est par lui aussi que les rassemblements populaires et même les offices religieux, l'enseignement et l'exercice de la loi - pourtant facteurs de discipline et de cohésion sociale - sont interdits (XXXII). Et l'on peut présumer alors du traumatisme que devaient représenter les enterrements effectués à la hâte et sans cérémonie (XXXV).

Le système d'approvisionnement doit être performant: une parcellisation ne fonctionne que si chaque partie peut subvenir à ses besoins. D'où l'importance, pendant toute la période précédant l'arrivée de la peste, de constituer des réserves suffisantes. L'article LXXIII du Règlement atteste qu'en décembre 1721, Genève est suffisamment pourvue de blé, de vin, de sel, etc. A l'exception de la viande, dont, pour des raisons évidentes, l'approvisionnement se fait aussi tard que possible, il n'y a guère que le bois à bâtir (point $7^{\circ}$ ) qui manque.

Enfin, le dispositif ne fonctionnera que policé, gourmé dans une gestion stricte qui en assurera le bon déroulement et en préviendra les abus. Conserver une vision détaillée de l'état de morbidité en ville (XXI et XLI) est essentiel au maintien de la politique sanitaire. Comme l'est aussi la propreté publique: plus question alors de transiger, tous les animaux doivent être abattus (XXXVIII) et les métiers considérés comme dangereux étroitement surveillés. C'est le cas de la boucherie et de la tannerie (XXXIX), avec leurs déchets facilement corruptibles. A ces points de gestion structurelle s'ajoute ce qu'aujourd'hui nous appellerions l'encadrement psychologique des pauvres malades, soit leur consolation morale, assumée par la compagnie des pasteurs (VI). Par ailleurs, un couvre-feu dès la tombée du jour (XXXVII) doit faciliter la tâche de surveillance des gardes et les travaux d'évacuation des corps malades. En contrepoint de l'atmosphère de stagna-

26 Aeg - Santé C1, f $100-101$. 
tion diurne, imposée par les barrières d'isolement, la nuit résonne du ballet des corbeaux et des marrons. Sur la lenteur du temps des quarantaines se plaque la folle course à la salubrité: on ne compte plus les articles qui enjoignent à une exécution aussi prompte que possible des travaux sanitaires.

Le Projet d'un règlement genevois donne par ailleurs une étrange impression de renfermement, presque d'autarcie: il n'est plus question d'importations de marchandises, de problèmes de frontières et d'intérêts commerciaux. La Chambre de la Santé prend la forme d'une administration policière. La ville se fige et se crispe sur elle-même. Le maintien de l'ordre se profile comme la seule échappatoire possible devant la peste. A tel point que même l'après de la contagion est organisé. Un nombre conséquent d'articles est consacré à la fin de la maladie, à la désinfection générale (LXVII, LXIX), au remboursement des frais (LXVI, etc.) ou encore aux orphelins qu'aurait engendrés la peste (LXVIII). Le financement des charges prohibitives occasionnées par le dispositif de protection sanitaire est d'ailleurs une préoccupation permanente, ce dont témoigne le point $1^{\circ}$ du dernier article. Lors de l'épidémie de 1636-1640, le Conseil dut intervenir plusieurs fois pour mettre fin aux nombreuses réclamations ayant trait à l'argent ${ }^{27}$.

Cette impression d'autarcie, même si elle émane du dispositif de parcellisation en soi, permet de pointer la spécificité de la situation genevoise. En effet, le Projet d'un règlement genevois réalise un plan de gestion de l'espace intra- et extra-muros; toute la campagne genevoise n'est évidemment pas aussi strictement délimitée que les terrains réquisitionnés pour la construction des cabanons sanitaires, mais on peut admettre que le Règlement permet de contrôler la quasi totalité de la population sur territoire genevois, et cela à partir d'une direction unique, la Chambre de la Santé. Un tel contrôle intégral s'avère beaucoup plus ardu pour, par exemple, Lausanne et le pays de Vaud; les campagnes y sont vastes et la répartition du financement des mesures contre la peste entre les autorités centrales de Berne et les autorités locales de Lausanne ne va pas de soi $^{28}$. L'avantage de la République de Genève tient dans son autonomie politique et dans les dimensions réduites de son étendue géographique. Ses possibilités d'action sont rapides et non entravées ou ralenties par l'administration des rapports entre un centre institutionnel et sa périphérie.

Cette exigence fondamentale d'ordre a son principe connexe, qui est l'utilité. Il s'agit de mettre à disposition tout ce qui sert et de rejeter ce qui est superflu. C'est ainsi qu'il faut emmagasiner les vivres de première nécessité et les médicaments, mais rejeter les denrées périssables, qu'il faut réqui-

27 Gautier 1888, 24.

28 Olivier 1939b, 617-618. 
sitionner du personnel soignant et des gardes, mais exclure les vagabonds et les étrangers. Le critère du rejet de l'inutile, facteur de désorganisation sociale, est permanent mais implicite dans le projet genevois. Dans son célèbre Traité de la Peste de 1721, adressée à «Messieurs les Docteurs Médecins qui composent le Corps de la Faculté de Médecine de Genève», corps auquel il appartient, Jean-Jaques Manget (1652-1742) recommande aux marchands de congédier la plupart de leurs serviteurs pour n'en conserver que ce qu'exige la plus stricte nécessité ${ }^{29}$. Et l'article $\mathrm{L}$ du modèle de règlement qu'il donne dans son traité, sorte de récapitulatif des bases sur lesquelles doit reposer la défense d'une cité contre la peste,rappelle que la première maxime à observer «est de faire sortir tout le peuple, en ne retenant que les personnes nécessaires $»^{30}$.

$\mathrm{Au}$ demeurant, les bases théoriques sur lesquelles est construite l'action sanitaire sont cohérentes. On pensait à l'époque que le venin pestilentiel, responsable de la transmission de la maladie, était tenace et subtil; s'incorporant à l'air, il était particulièrement bien retenu par les corps réputés spongieux ou poreux, tels la laine et les plumes ${ }^{31}$. En outre, la crasse et la putréfaction favorisaient son développement. Ce sont ces idées qui motivent la méfiance envers les bouchers et les tanneurs, envers les lits et les hardes des malades, qui justifient les fréquents changements d'habits et qui légitiment les appels à la propreté32. Au contraire, la toile cirée qui figure dans la liste d'approvisionnement du dernier article a la réputation de n'offrir que peu de prise au venin pestilent; Manget en préconise l'emploi pour les habits du personnel fréquentant les lieux infectés ${ }^{33}$, tandis que Benoît Textor (?-1560) en recommande l'usage comme isolant pour les habitations privées $^{34}$. On trouve également de nombreuses références au parfumage ou à la purification dans ce Règlement. Cette pratique, classique de la lutte contre la peste, consiste en des fumigations d'herbes odorantes, souvent âcres et puantes «comme assa foetida, vieux souliers, poil de porc \& de bouc» ${ }^{35}$, destinées à «tuer» le venin pestilent. L'épreuve mentionnée à l'article LXX doit servir de test: Manget explique qu'elle consiste à

29 Manget 1721, t. 1,130.

30 Manget 1721, t. 1,138.

31 De La Breuille 1641, 20 ss.

32 Les pratiques changent toutefois moins vite que les théories; un geste prophylactique ou thérapeutique qui perdure pendant plusieurs siècles peut procéder de logiques différentes (Wenger 2001, 76).

33 Manget 1721, t. 1, 171.

34 Textor $1551,39$.

35 Canadelle 1636, 64. 
prendre quelques pains tous chauds venants du four, \& les ouvrir, puis les atacher au bout d'une picque, ou halebarde, en les laissant aux Chambres, ou l'infection aura été plus grande, après avoir bien fermé les fenêtres, durant vint-quatre heures: car s'il reste encore du venin, le pain se pourrit, changeant de couleur \& de gout, il le faut enterrer soigneusement sans le toucher: Que s'il ne change pas, \& ne reçoit aucune altération, la désinfection est parfaite. L'autre expérience qu'ils aportent, est, d'atacher au bout d'un bâton, de la chair de Mouton, ou autre qui soit fraiche, [etc.]. ${ }^{36}$

Le Règlement genevois met donc en place un dispositif qui réduplique dans la cité ce qui se passe au niveau interurbain: de la même manière que les villes et les régions sont séparées par des cordons sanitaires, Genève est parcellisée. Mais ce système connaît ses limites. Les marrons, engagés le temps de l'épidémie pour le nettoyage et le débarrassage des maisons contaminées, sont craints par la population autant qu'ils sont essentiels au dispositif de lutte contre la peste. On les soupçonne souvent d'«engraisser» les serrures et les seuils des portes avec du venin pestilent pour conserver leur activité et s'enrichir impudemment. Dans le passé, Genève n'a pas manqué d'exécuter certains de ces supposés engraisseurs ${ }^{37}$. L'article LXVIII atteste que l'inventaire des meubles du domicile d'un mort de peste, en prévision de l'établissement de l'héritage, se fait à un greffier situé dans la rue, par des marrons - traditionnellement soupçonnés de vol - qui pénètrent dans la pièce.

En l'absence d'une théorie et d'une thérapeutique médicales réellement opératoires, les villes d'Ancien Régime menacées de peste n'ont de choix que la discipline - ce en quoi le Règlement genevois est tout à fait représentatif de son époque. Dans le terme sanitaire, il entrait alors des acceptions aujourd'hui perdues. Jaques Chenaud (1654-1741), l'un des deux représentants du corps médical dans la Chambre de la santé pendant la peste de Marseille, l'illustre bien lorsque le 2 septembre 1720, il requiert «que l'on mette des potences aux portes, comme on fait ailleurs dans des conjonctures semblables, pour détourner les personnes suspectes qui pourraient s'introduire par adresse ${ }^{38}$. Une proposition répressive et bien peu médicale qui montre tout ce qui entre dans la notion de protection du bien public en temps de peste.

«L'intérêt des autorités administratives pour la peste est le fait capital, le point de départ essentiel de toutes les réglementations administratives ultérieures dans le domaine de l'hygiène publique.» ${ }^{39}$ La peste est directement à l'origine de la création de nombreux conseils de santé.Le cas de Berne 
est explicite: «C'est le 29 avril 1650 que pour la première fois les affaires sanitaires sont remises par le Petit Conseil à un mandataire spécial. [...] Le 14 novembre 1679, le Sénat décide d'instituer une chambre spéciale. [...] Son nom de Conseil de Santé (Sanitätsrat) date du 18 octobre 1709, en ce sens que la commission fut alors réorganisée.» ${ }^{40}$ Que ce soit en 1679 ou en 1709, ce qui engage le gouvernement bernois à la création du Conseil de Santé, c'est la crainte de la contagion, c'est-à-dire de la peste ${ }^{41}$. Les conseils fonctionnent comme un réseau de surveillance mutuelle; Bâle et Lucerne instaurent donc les leurs également au début du XVIII ${ }^{\mathrm{e}}$ siècle $^{42}$.

«Despotisme médical et absolutisme éclairé se prêtent ici mutuel secours pour une œuvre qui est à la fois d'assistance et de discipline. [...] Soigner, c'est diriger. ${ }^{43}$ Cette alliance directive entre la science et le politique culminera au XVIII ${ }^{\mathrm{e}}$ siècle dans le programme d'action de la Société Royale de Médecine de Paris (créée en 1776), entièrement fondé sur la figure du médecin des épidémies ${ }^{44}$. Or, les réactions face à la peste de Marseille constituent déjà un exemple de cette alliance, sinon par le recours dès le mois d'août 1720 à l'armée pour assurer les cordons sanitaires (on utilise en particulier les troupes nombreuses disponibles dans le sud de la France après la guerre d'Espagne de 1719-1720) ${ }^{45}$, du moins par la concertation au niveau national qui préside à leur établissement.

$\mathrm{Au}$ fil des épidémies successives et des informations échangées par les instances sanitaires des différentes régions incriminées, le but est donc de prévenir les dégâts en instaurant un contrôle pragmatique et rationnel de la situation de crise - le Règlement genevois en témoigne de manière exemplaire. Cela d'autant plus qu'il ne faut pas trop attendre des «Médecins prudens \& experimentez» qui, comme le rappelle Manget, sont souvent précautionneusement «retirez» ${ }^{46}$ en un lieu sûr où ils n'entretiendront que peu de contact avec les malades. Bien plus, devant l'incertitude de la maladie et en l'absence de mesures thérapeutiques réellement opératoires, c'est vers le passé, parfois lointain, et les mesures instaurées lors des précédents ravages épidémiques que l'on se tourne dans l'espoir d'y puiser des solutions pour la situation présente.

40 Olivier 1939b, 1.

41 Olivier 1939b, 3.

42 Olivier 1939b, 8.

43 Roche 1988, 328.

44 Daniel Roche a souligné la manière dont, dans la cinquantaine d'éloges qu'il prononce à la Société Royale de Médecine entre 1776 et 1789, Vicq d'Azyr construit cet idéal du médecin des épidémies (Roche 1988, 328).

45 Panzac 1986, $60 \mathrm{~s}$.

46 Manget 1721, t. $1,81 \& 202$. 


\author{
Abréviations \\ Aeg = Archives d'Etat de Genève \\ $\mathrm{PH}=$ Aeg, Pièces Historiques \\ $\mathrm{RC}=$ Aeg, Registres des Conseils \\ Santé $=$ Aeg, Procès-verbaux de la Chambre de la Santé A5, 44
}

\title{
Bibliographie
}

Biraben, Jean-Noël, Les hommes et la peste en France et dans les pays européens et méditerranéens, 2 tomes (Paris 1975)

Canadelle, Moyse, Petit traicté et familier de la peste (Genève 1636)

Carpentier, Elisabeth, Une ville devant la peste. Orvieto et la Peste Noire de 1346 (Paris 1962)

Chaponnière, Jean-Jacques/Louis Sordet (éds), Des hôpitaux de Genève avant la Réformation (Genève 1844)

Cipolla, Carlo Maria, Contre un ennemi invisible. Epidémies et structures sanitaires en Italie de la Renaissance au XVII siècle (Paris 1992)

De La Breuille, Jean-Louis, Traicté de la contagion et de ses remedes (Genève 1641)

Gaffarel, Paul, La Peste de 1720 à Marseille et en France (Paris 1911)

Gautier, Léon, La dernière peste de Genève: 1636-1640 (M. D. G. 23,1-61) (Genève 1888)

- La médecine à Genève jusqu'à la fin du XVIII siècle (Genève 1905)

Manget, Jean-Jaques, Traité de la peste, recueilli, des meilleurs auteurs anciens \& modernes, et enrichi de remarques \& observations theoriques \& pratiques, 2 tomes (Genève 1721)

Olivier, Eugène, Médecine et santé dans le Pays de Vaud. Des origines à la fin du XVII siècle, 2 tomes (Lausanne 1939a)

- Médecine et santé dans le Pays de Vaud, 1675-1798, 2 tomes (Lausanne 1939b)

Panzac, Daniel, Quarantaines et lazarets. L'Europe et la peste d'Orient (Aix-en-Provence 1986)

Perrenoud, Alfred, La population de Genève du seizième au début du dix-neuvième siècle (Genève Paris 1979)

Roche, Daniel, Les Républicains des lettres. Gens de culture et Lumières au XVIII siècle (en particulier le chap. XIII: «Médecins et Lumières au XVIII ${ }^{e}$ siècle: talents, raison et sacrifice») (Paris 1988)

Slack, Paul, The Impact of plague in Tudor and Stuart England (London [etc.] 1985)

Textor, Benoît, Traicté de la peste divisé en deux livres, l'un de la preservation, l'autre de la cure d'icelle (1551)

Wenger, Alexandre, «Genève et la peste de Marseille de 1720», Medicina \& Storia 1 (2001)

\section{Annexe}

Transcription du «Projet d'un Règlement général», imprimé à Genève en décembre 1721 (Aeg, PH 4455 pièce $\mathrm{n}^{\circ}$ 9). 
[p. 1]

\section{P R O J E T \\ D'U N R E G L E M E N T G E N E R A L,}

Pour l'ordre, qui devra être observé en cette Ville, au cas qu'elle vint à être afligée de la Peste; ce qu'il plaise à Dieu de détourner par sa grande misericorde.

Ledit Réglement projetté par la Chambre de la Santé,établie en cette Ville en l'Année 1721. pour être proposé à Nos SeIgneurs Du Petit et GRAND Conseil, \& y recevoir force de Loi.

\section{A R T I C L E P R E M I E R.}

I A Chambre de la Santé, telle qu'elle a été établie cette année, sera de plus fort confirmée dans ses fonctions; sauf à augmenter le nombre des Membres qui la composent, suivant la circonstance des temps.

II.

La Chambre s'assemblera tous les jours à la maison de Ville à 8 . heures du matin; \& si le mal alloit en augmentant; elle devra s'assembler de relevée à une heure après midi, pour être à portée de pourvoir à tous les Cas, qui peuvent survenir d'un moment à l'autre.

III.

Les Conseils procéderont à l'Election d'un Capitaine ou Commissaire Géneral de la Santè, qui reçevra immédiatement les ordres de la Chambre, \& sera chargé de les faire exécuter avec fidélité \& promtitude; Il aura 8. Archers ou Huissiers à sa disposition.

IV.

Les Conseils nommeront aussi huit Commissaires des Quartiers, auxquels on distribuera les differens Quartiers de la Ville, le plus également qu'il sera possible; sauf à augmenter le nombre des dits Commissaires suivant le besoin.

V.

La Chambre établira trois Directeurs; savoir deux pour les Infirmeries \& un pour les Convalescens; Elle établira aussi les Infirmiers \& Infirmiéres, Pourvoyeurs, Chartiers, Corbeaux blancs, Corbeaux noirs, en telle quantité que le besoin le demandera.

VI.

La Vénerable Compagnie choisira de son Corps deux Spectables Pasteurs, pour la consolation des pauvres Malades.

[p. 2]

VII.

Messieurs de la Faculté de Médecine choisiront aussi dans leur Corps deux Médecins, deux Apoticaires, quatre Fraters Apoticaires; quatre Chirurgiens; huit Fraters Chirurgiens, pour le service des Malades; sauf à augmenter le nombre suivant le besoin.

VIII.

Les Fraters Chirurgiens qu'on aura reconnu s'étre distingué dans le service des Malades, seront gratifiez de la Bourgeoisie \& de la Maîtrise.

IX.

Le Grand Pré de la Coulouvreniere \& le Clos du Cimetiére pourront servir pour y établir les Infirmeries des Pauvres, en y faisant construire un nombre suffisant de Cabanes de bois, separées les unes des autres. 
$\mathrm{X}$.

L'Espace de Terrain confiné comme suit; Le chemin tendant au petit Pleinpalais du Levant; Le petit Pleinpalais \& le chemin tendant à la Coulouvreniere du Vent; Le Fleuve du Rhône du Couchant, \& le Glacis de la Ville de Bise; Tout cet espace, \& les maisons qu'il renferme pourra servir pour les infirmeries des personnes moyennées.

\section{XI.}

Le Grand Pré du Sr. La Combe pourra servir pour les Pauvres Convalescens, en y faisant construire des Baraques.

XII.

L'Espace confiné comme suit; Le chemin tendant au petit Pleinpalais du Couchant; Le Pleinpalais de Bise; Le sentier tendant au Jardin du Sr. Michel Mollet du Levant; Le dit Jardin Mollet du Vent; Tout cet espace \& les maisons qu'il renferme pourra servir pour les Convalescens moyennez.

\section{XIII.}

Toutes les Prairies, dites les Savoises, pourront servir pour les Pauvres suspects envoyez en Quarantaine, en y faisant construire des Baraques.

\section{XIV.}

Toutes les Maisons comprises dans la Partie Méridionale de Pleinpalais, jusques au chemin de la Tour d'Arve, serviront pour les suspects moyennez, envoyez en Quarantaine.

\section{XV.}

Les Cuisines des Infirmeries pourront être établies, dans la Maison de la Coulouvreniere, \& dans Celle de l'Hôpital des Pestiferez. Celle des Pauvres Convalescens dans la Maison du Sr. La Combe, \& Celle des Pauvres Suspects, dans la Maison qui paroitra la plus commode proche de leur Quartier.

\section{XVI.}

Les malades Convalescens \& suspects moyennez, feront leur cuisine dans les maisons qu'ils occuperont, \& se feront servir par leurs propres Domestiques.

\section{XVII.}

Les personnes emploïées aux Infirmeries, logeront dans la Maison de la Coulouvreniere \& dans celle ditte des Volontaires; \& ceux qui serviront les Convalescents, logeront dans la maison du Sr. La Combe.

XVIII.

Les Corbeaux \& Charretiers destinez aux transport des Malades \& Cadavres, seront logez dans les Capites, qui environnent le Cimetiére.

XIX.

Les Nouveaux Fossez comblez sour la Treille, serviront pour faire camper neuf Compagnies de la Garnison, \& les trois autres pourront camper dans les Prez de Clébergue, au Quartier de Saint Gervais.

\section{XX.}

Au cas qu'on veuille faire camper une partie des Hanitans pendant la désinfection generale, si elle est jugée nécessaire; Le grand Pré de Mr. le Conseiller Franconis \& le Pleinpalais paroissent les plus propres.

\section{XXI.}

Les Commissaires des Quartiers feront deux fois par jour la ronde de leur Quartier, pour s'informer des Nouveaux malades, qui pourroient étre au Cas; Ils en rapporteront la liste au 
Commissaire Général de la Santé, qui les fera transporter sur le champ avec leur lit dans des chariots couverts, conduits par les Corbeaux blancs aux Infirmeries; Si c'est une personne pauvre, dans l'Infirmerie des Pauvres; \& si c'est une personne moyennée, dans celle des moyennez, ce, sans aucune distinction.

[p. 4]

XXII.

Lorsqu'une personne sera morte subitement du mal contagieux dans une maison; les Parents ou Domestiques du mort dévaleront le cadavre envelopé dans un drap à la porte de la ruë, lequel sera envlevé par les Corbeaux noirs, le plûtôt qu'il sera possible, à la diligence du Commissaire de Quartier.

\section{XXIII.}

Les Habitants d'une Maison, soit Proprietaires, soit Inquilins, dans laquelle sera mort ou tombé malade quelque personne au Cas, resteront sequestrez dans leur maison, pour y faire Quarantaine, toutes les avenuës de la dite Maison seront cancelées, l'on appliquera à la porte une Marque, pour faire conoître qu'elle est infectée, laquelle y restera jusques-à-ce que la Chambre de la Santé ait ordonné la purification de la dite Maison.

\section{XXIV.}

La Chambre pourvoira à ce qu'il soit fourni des Vivres aux Habitans des Maisons séquestrées, \& ce suivant les états qui en seront donnez par les Commissaires des Quartiers.

XXV.

Les Médecins, Apotiquaires \& Chirurgiens, qui auront été voir quelques malades, qui se trouveront frappez de la Peste, se séquestreront dans leur maison pendant dix jours, dès la dernière visite, \& il leur sera mandé de se parfumer \& changer d'habits avant que d'être liberez.

\section{XXVI.}

Les Personnes qui seront entrées \& resorties promtement d'une maison infectée, seront pareillement séquestrées pour 15 . jours dans leur maison, \& seront obligées de se parfumer \& de changer d'habits avant que de sortir.

\section{XXVII.}

Ceux qui auront servi un malade, quelques jours avant que d'avoir connu la maladie, seront aussi renfermez pendant trente jours, se parfumeront \& changeront d'habits.

XXVIII.

Ceux qui auront servi les Malades moyennez dans les infirmeries, resteront sequestrez pendant quarante jours, pendant lesquels ils devront faire les lessives \& essors nécessaires, seront parfumez \& changeront d'habits avant que d'étre liberez.

[p. 5]

\section{XXIX.}

La Chambre de la Santé jugera des resserremens \& des libérations des personnes; ayant égard aux temps, aux lieux, aux commoditez des Maisons \& autres circonstances.

\section{XXX.}

Elle aura aussi le pouvoir de condanner à des amendes ceux qui contreviendront aux Ordonnances, \& le deniers en provenans, seront raportez au Trésorier de la Chambre, \& seront employez à la dépense publique. 
XXXI.

Il sera tenu un Compte exact des frais que la Chambre fera pour les Malades moyennez, \& pour la nourriture des personnes moyennées séquestrées, \& il en sera dressé une parcelle à chacun, pour en exiger le remboursement, \& les deniers en provenans, seront pareillement remis entre les mains du Trésorier de la Chambre.

\section{XXXII.}

Les Temples, les Ecoles \& les Cours de Justice, resteront fermées pendant le temps de la Contagion, \& ne seront rouvertes que par l'ordre des Conseils sur le rapport que la Chambre leur fera de l'état de Santé publique.

\section{XXXIII}

Les Commissaires des Quartiers, Archers, Corbeaux, Charretiers, \& généralement tous ceux qui s'approcheront par le devoir de leurs charges des morts ou malades au Cas, seront regardez comme suspects, \& ne marcheront jamais sans leur Robe de toile cirée avec une marque de couleur dessus, \& une baguette blanche de 4. pieds à la main.

\section{XXXIV.}

Les Corbeaux ou Charretiers employez à la conduite des Malades ou Cadavres, porteront une Clochette, pour averti ceux qui se trouveront à leur passage, de se retirer.

\section{XXXV.}

Les Personnes qui mourront de maladie ordinaire, non suspecte, seront enterrées sans cérémonie dans le lieu à ce destiné, \& l'on assignera pour cet effet une place hors de chaque Porte. [p. 6]

\section{XXXVI}

L'endroit, dit, derriére les Cibes, a été jugé le lieu le plus convenable pour l'inhumation des Cadavres morts de Peste.

\section{XXXVII}

Il sera ordonné à tous les habitans, de quelle qualité \& condition qu'ils soient, de rentrer $\&$ de se renfermer dans leurs Maisons après le Soleil couché; sauf les Employez, ou ceux qui en auront obtenu la permission de la Chambre.

\section{XXXVIII.}

L'on réitérera les défenses de nourrir des Pigeons, Canards \& Oisons, \& l'on fera tuer tous les Chiens \& les Chats.

\section{XXXIX}

Les Bouchers devront tuer dans un quartier éloignée de toute habitation; \& les Taneurs porteront leur fabrique hors la Ville.

XL.

Messieurs les Médecins, Apotiquaires \& Chirurgiens, seront mis sous le serment de ne point s'absenter de la Ville pendant la Contagion, \& de déclarer la nature du mal des Malades qu'ils verront.

\section{XLI.}

Il sera aussi fait une publication expresse pour obliger tous les particuliers, de quelle qualité $\&$ condition qu'ils soient, de déclarer au Commissaire du Quartier les Malades qu'ils pourroient avoir dans leurs Maisons, \& la nature de leur maladie. 
XLII.

Défenses très expresses seront faites aux Ministres, Médecins, Apotiquaires \& Chirurgiens, destinez pour le soulâgement des Malades au Cas, de voir ni fréquenter aucun autre Malade.

\section{XLIII.}

La Peste étant déclarée; on donnera un terme de 8. jours à ceux qui voudront faire retirer dans leurs Campagnes, leurs familles \& leurs effets, passé lequel tems, il ne leur sera plus permis de les faire sortir.

[p. 7]

\section{XLIV.}

Messieurs Les Docteurs Médecins devront faire une Ordonnance, portant la maniére dont chaque personne saine devra se parfumer, tant sa personne, que son habitation; Comme encore les préservatifs, qu'il conviendra de prendre pour se garentir du mal. Ils ordonneront aussi les précautions \& les préservatifs, dont se devront servir les personnes employées pour le service des Malades au Cas.

XLV.

Les Lieux destinez pour les Infirmeries, tant des Pauvres, que des Moyennez, seront entourés de Palissades ou Palins, en y pratiquant une seule Barriere, à laquelle on établira une Garde.

XLVI.

Défenses à peine de la vie seront faites, d'entrer du quartier des Infirmeries, ou d'en sortir, par un autre endroit, que par la Barriere.

\section{XLVII.}

Les Corbeaux blancs \& Charretiers, qui transporteront les pauvres malades aux Cabanes, y transporteront en même temps leurs lits, s'ils en ont, \& s'ils n'en n'ont point, l'Hôpital leur en fournira.

\section{XLVIII.}

Les Linges, Vivres, Drogues, Savon, Parfums, Médicamens pour les Infirmeries, seront fournis par l'Hôpital Général.

XLIX.

Il sera envoyé chaque jour de l'Hôpital à la barriere des Infirmeries, la quantité de vivres nécessaire, suivant que la Chambre l'aura réglé par personne, \& ce, sur le Certificat du Directeur des Infirmeries, attesté par le Ministre, contenant le nombre des malades.

L.

Il envoyera aussi la quantité des drogues \&c. nécessaire, sur le Certificat du Médecin. [p. 8]

LI.

Le Directeur des Infirmeries tiendra un Régître, où il enrégîtrera de jour à jour le Nom \& Surnom des Malades entrez aux Cabanes ou au Quartier des moyennez; l'heure de leur entrée, celle de leur sortie, ou de leur mort.

\section{LII.}

Il fera enlever avec promtitude les Cadavres des morts aux Infirmeries par les Corbeaux noirs, qu'il aura à sa disposition, \& qui les enterreront au lieu qui leur sera marqué. 
LIII.

Les Meubles ou le Lit du mort seront bruslez à l'entrée de la Cabane; laquelle sera parfumée du parfum fort, \& restera fermée pendant huit jours, s'il est possible, avant que d'y mettre au autre malade. Les Lits \& Hardes, qui auront servi à ceux qui seront morts subitement dans la Ville, seront pareillement brûlez.

\section{LIV.}

Le Directeur numerotera toutes des dittes Cabanes, \& tiendra un Régître éxact de leur purification.

LV.

Les personnes moyennées, affligées du mal, \& qui seront transportées dans le lieu qui leur sera assigné; auront la liberté de faire porte avec eux la quantité de meubles \& autres choses nécessaires à la vie, qu'ils jugeront à propos; Comme encore de se faire servir par tel nombre de domestiques qu'ils souhaiteront, ou personnes de leur famille; à condition que les dittes personnes \& domestiques, seront regardez comme suspects, n'entreront point dans laVille, \& ne communiqueront point avec les maisons voisines.

LVI.

Les Malades moyennez se feront aporter journellement de la Ville tout ce dont ils auront besoin; en observant de le faire aporter à la barriére, d'où on le jettera en dedans aux Domestiques suspects, qui le viendront prendre.

LVII.

Toutes les personnes renfermées dans l'Enclos des Infirmeries, seront reputées suspectes, $\&$ porteront une baguette blanche de 4. pieds à la main.

[p. 9]

LVIII.

Le Directeur des Convalescens tiendra un Régître exact de leur entree en Convalescence, $\&$ de leur sortie du quartier; Et les vivres pour les Pauvres, seront fournis par l'Hôpital Géneral, sur le Certificat qu'il donnera du nombre desdits Convalescens.

\section{LIX.}

Les Convalescens moyennez se feront aporter les Vivres de la Ville, dans le quartier qui leur sera assigné.

LX.

Les Pauvres Suspects ou Quarantenaires, se rendront dans le lieu qui leur sera destiné, \& y seront nourris aux dépens du Public.

\section{LXI.}

Les Personnes de considération, qui seront dans le Cas de la quarantaine, \& qui voudront choisir un lieu hors de la Ville pour s'y retirer, le pourront faire par permission de la Chambre, en leur donnant une ou deux Gardes, qui seront payées \& nourries à leurs dépens.

\section{LXII.}

Les Convalescens \& Quarantenaire pourront rentrer dans la Ville, en aportant un Certificat du Directeur; Comme ils ont fini le temps de leur quarantaine présigé par la Chambre, changé d'habits, \& passez par les parfums ordonnez. 
LXIII.

A l'égard des pauvres Malades de la Campagne; Ceux de la Banlieuë pourront étre conduits aux Infirmeries; \& à l'égard des Villages écartez; la Chambre pourvoira autant qu'il lui sera possible, châque Village, d'un Frater Chirurgien, auquel on fournira les Drogues \& Médicaments nécessaires.

\section{LXIV.}

Il sera aussi établi un Notable ou deux à châque Village, pour Inspecteurs de Santé; auxquels on recommandera de faire séquestrer diligenment les malades d'avec les Sains, \& de faire enterrer les Cadavres avec promtitude.

LXV.

Si lesdits Villages manquent de Bled; il leur en sera fourni par l'Hopital Géneral. [p. 10]

\section{LXVI.}

L'Hôpital Géneral sera privilégié pour le remboursement des frais qu'il aura fait pour le service \& assistance des Personnes mortes de Peste.

\section{LXVII.}

S'il est jugé nécessaire après la Contagion, de faire une Désinfection génerale de la Ville, la Chambre jettera les yeux sur des personnes expertes en ce fait; traittera avec elles au meilleur conte possible; \& veillera à ce que la ditte désinfection se fasse suivant ce qu'elle aura ordonné.

\section{LXVIII.}

Si les Morts de Peste laissent des Hoiries jacents, ou des Pupilles \& Mineurs, l'Inventaire des meubles \& effets se fera sommairement, lors de la désinfection, par le Sr. Auditeur du Quartier, avec son Gréfier, à la révélation des Marrons ou Dessouillonneurs, qui se spécifieront par la fenêtre, le Sr. Auditeur \& son Gréfier étans à la Ruë.

\section{LXIX.}

Tous les Meubles, Vaisselle, Denrées, Marchandises \& Monnoye existans dans une Maison infectée, de quelque étenduë qu'elle soit, seront sujets à la désinfection.

\section{LXX.}

Les Marques apliquées sur les Portes des Maisons infectées seront enlevées après l'épreuve de la désinfection, faite au moyen du pain chaud, ou de la chair cruë, comme cela s'observe en pareil Cas.

\section{LXXI.}

On assignera un lieu propre pour faire camper les Habitans des maisons infectées, \& ils ne rentreront qu'après les termes qui leur seront prescrits par la Chambre.

\section{LXXII.}

La Chambre de la Santé réglera les Gages de toutes les personnes stipendiées, qu'elle employera à l'occasion de la Contagion.

[p. 11] 


\section{A P P R O V I S I O N N E M E N T}

\section{Des choses nécessaires à la Ville, en cas de Contagion.}

De l'Argent.

Du Bled.

Du Vin.

Du Sel.

Viande de Boucherie.

Vinaigre.

Eau de vie.

Epiceries.

Drogues.

Bois Aromatiques.

Bois à Bâtir.

Bois à Brûler.

Palissades ou Palins.

Chaux vive.

Linge neuf \& vieux.

Toile Cirée.

Ras de Saint Maur ou Gros de Tour.
Charbon.

Braise.

Savon.

Poudre à Canon.

Graine de Genève.

Tabac.

Fil de fer.

Paniers d'ozier.

Huiles \& Suif.

Clouterie.

Du Ris.

Du Fromage.

\section{La Chambre ayant pris en considération les articles ci dessu, a fait les Réflexions suivantes.}

$1^{\circ}$. Par raport à l'Argent; Les Seigneurs de la Chambre des Comptes seront priez de mettre en reserve, au moins la somme de cent mille Ecus, uniquement destinez pour les besoins \& frais occasionnez par la Contagion.

$2^{\circ}$. A l'égard du Bled, la Chambre estime que la Ville en est suffisanment pourvüe, tant par celui qui est dans les Gréniers Publics, que par les Provisions extraordinaires que les Particuliers ont fait en ces derniers temps.

3․ A l'égard du Vin, du Vinaigre, de l'Eau de vie, des Epiceries, du vieux Linge, du Ras de Saint Maur, Gros de Tours, du Savon, de la Poudre à Canon, Tabac, Fil de fer, Paniers d'ozier, Huiles \& Suif, Clouterie; Il y en a suffisanment dans la Ville.

4. A l'égard du Sel, on conte aussi sur celui qui est dans les Magazins Publics.

$5^{\circ}$. A l'égard de la Viande de Boucherie, Bois à brûler, Chaux, Charbon, Braise, Graine de Genèvre, Ris, Fromage; La Chambre établira des Personnes afidées dans le déhors, qui auront soin d'en faire les provisions, \& de les faire conduire aux Barrieres.

[p. 12]

6․ A l'égard des Drogues \& Bois Aromatiques, Messieurs les Marchands Droguistes \& Apotiquaires ont été exhortez depuis plusieurs mois, de s'en fournir abondanment.

$7^{\circ}$. Pour le Bois à bâtir, Les Seigneurs de la Chambre des Comptes seront priez de donner leurs ordres, pour qu'il leur soit fourni jusqu'à la concurrence de six mille douzaines de Planches, \& le reste à proportion.

8. A l'égard du Linge neuf \& de la Toile cirée; l'Hôpital a donné les ordres, pour en avoir une bonne provision. 